\title{
Comparison of Effectiveness between Abdominal Vibration Stimulation and Walking Exercise for Bowel Cleansing before Therapeutic Colonoscopy
}

\author{
Choong-Kyun Noh ${ }^{1}$, In Sung Kim ${ }^{1}$, Gil Ho Lee ${ }^{1}$, Jin Woong Park ${ }^{1}$, Eunyoung Lee ${ }^{2}$, Bumhee Park ${ }^{2}$, Hye Jeon Hong ${ }^{3}$, Sun Gyo \\ $\mathrm{Lim}^{1}$, Sung Jae Shin ${ }^{1}$, Jin Hong Kim ${ }^{1}$, and Kee Myung Lee ${ }^{1}$ \\ Departments of ${ }^{1}$ Gastroenterology and ${ }^{2}$ Biomedical Informatics, Ajou University School of Medicine, and ${ }^{3}$ General Ward Nursing Unit, Ajou \\ University Medical Center, Suwon, Korea
}

Background/Aims: Adequate bowel preparation is important for successful colonoscopy. We aimed to evaluate the clinical feasibility and effectiveness of abdominal vibration stimulation in bowel preparation before therapeutic colonoscopy. Methods: A single center, prospective, randomized, investigator-blinded study was performed between January 2016 and December 2016. Patients for therapeutic colonoscopy were prospectively enrolled and assigned to either the vibrator group or walking group. Patients who refused to participate in this study as part of the experimental group consented to register in the control group instead. During the preparation period, patients assigned to the walking group walked $\geq 3,000$ steps, whereas those assigned to the vibrator group received abdominal vibrator stimulation and restricted walking. All patients received the same colon cleansing regimen: 4-L split-dose polyethylene glycol (PEG) solution. Results: Three hundred patients who received PEG solution for therapeutic colonoscopy were finally enrolled in this study ( $n=100$ per group). Bowel cleansing with abdominal vibration stimulation showed almost similar results to that with walking exercise (Boston Bowel Preparation Scale score for the entire colon: vibrator vs walking vs control, $7.38 \pm 1.55$ vs $7.39 \pm 1.55$ vs $6.17 \pm 1.15, p<0.001)$. There were no significant differences between the vibrator group and walking group regarding instances of diarrhea after taking PEG, time to first diarrhea after taking PEG, total procedure time, and patient satisfaction. Conclusions: This study indicates that, compared with conventional walking exercise, abdominal vibration stimulation achieved similar rates of bowel cleansing adequacy and colonoscopy success without compromising safety or patient satisfaction. (Gut Liver 2020;14:468-476)
Key Words: Colonoscopy; Bowel cleansing; Abdominal vibration; Stimulation; Walking exercise

\section{INTRODUCTION}

Colon cleansing is very important to ensure the diagnostic accuracy and therapeutic safety of colonoscopy. Poor bowel preparation is associated with a risk of interval cancer due to missed lesions, earlier-than-necessary recall for surveillance, and serious adverse events such as intestinal perforation. ${ }^{1-3}$ Additionally, poor bowel preparation prolongs procedure time, ${ }^{4}$ which increases the need for sedative medication and often results in poor patient compliance and decreased patient satisfaction.

Walking exercise is known to improve bowel clearance and reduce the discomfort associated with nausea and abdominal pain during bowel preparation for colonoscopy. ${ }^{5}$ A previous study recommends walking at least 3,000 steps during bowel preparation. ${ }^{5}$ However, walking exercise can be uncomfortable, difficult, or even impossible in some patients. Therefore, it is desirable to develop a method that provides a similar effect as that of walking exercise, but in a more convenient manner for patients unable or unwilling to perform walking exercise.

Abdominal massage and whole-body vibration therapy have been shown to help improve symptoms in patients with severe constipation. ${ }^{6,7}$ Abdominal vibration stimulation has been reported to increase motility of the gastrointestinal (GI) tract, to reduce colon transit time, and to reduce constipation in older patients. In particular, physical massage of the abdomen increases bowel movement resulting in reduced constipation symptom, alleviates abdominal distension, and improves fecal

Correspondence to: Kee Myung Lee

Department of Gastroenterology, Ajou University School of Medicine, 164 World cup-ro, Yeongtong-gu, Suwon 16499, Korea

Tel: +82-31-219-5119, Fax: +82-31-219-5119, E-mail: lkm5104@ajou.ac.kr

Received on June 10, 2019. Revised on August 2, 2019. Accepted on August 19, 2019. Published online December $12,2019$. pISSN 1976-2283 eISSN 2005-1212 https://doi.org/10.5009/gnl19199

(a) This is an Open Access article distributed under the terms of the Creative Commons Attribution Non-Commercial License (http://creativecommons.org/licenses/by-nc/4.0) which permits unrestricted non-commercial use, distribution, and reproduction in any medium, provided the original work is properly cited. 
incontinence. $^{8,9}$

We hypothesized that abdominal vibration stimulation may improve bowel cleansing in preparation for colonoscopy. However, to date, no trial has evaluated the effect of abdominal vibration stimulation on colonic cleansing for colonoscopy. Therefore, we designed and conducted a prospective study to compare the bowel cleansing effect, clinical feasibility, and safety of abdominal vibration stimulation for bowel preparation.

\section{MATERIALS AND METHODS}

\section{Patients and study design}

This study was performed as a prospective, randomized, investigator-blinded, single-center study conducted between January 2016 and December 2016. All patients gave their written informed consent for participation before enrollment. The study protocol was approved by our institution's review board and ethics committee (approval number: AJIRBMED-MDB-15-381), and was conducted in agreement with established guidelines. The trial was registered to the Clinical Research Information Service (cris.nih.go.kr, approval number: KCT0002432).

All patients were originally managed at a local clinic, where they were diagnosed as having colonic polyps and were referred to our outpatient clinic for removal. The eligibility criteria were as follows: age, 20 to 80 years; undergoing therapeutic endoscopy such as endoscopic mucosal resection or polypectomy; relatively good health, reflected in an American Society of Anesthesiologists physical status of 1 or 2 . The reason we enrolled only patients who underwent endoscopic mucosal resection or polypectomy was because the participating nurses could closely observe whether the vibrator was used properly in these patients. Exclusion criteria were as follows: suspected colon obstruction; history of heart failure, renal failure, or severe dyspnea; bleeding tendency, reflected as international normalized ratio $>1.5$ or platelet count $<50,000$ cells $/ \mu \mathrm{L}$; allergy to polyethylene glycol (PEG); surgical history of gastrectomy or colonic resection; conditions associated with pronounced mucosal damage, such as progressive colonic dilatation or toxic megacolon; pregnancy; dementia; and inability to follow the study protocol.

Patients who refused to participate in the study as part of the experimental group consented to register in the control group instead. Then, patients who consented to participate this study were randomized to two groups (1:1 arranged to vibrator group and walking group, computer-generated random list). The allocation sequence was concealed and assigned only at the enrollment. After obtaining informed consent before registration, the researcher explained the research design and method to the patient (Fig. 1).

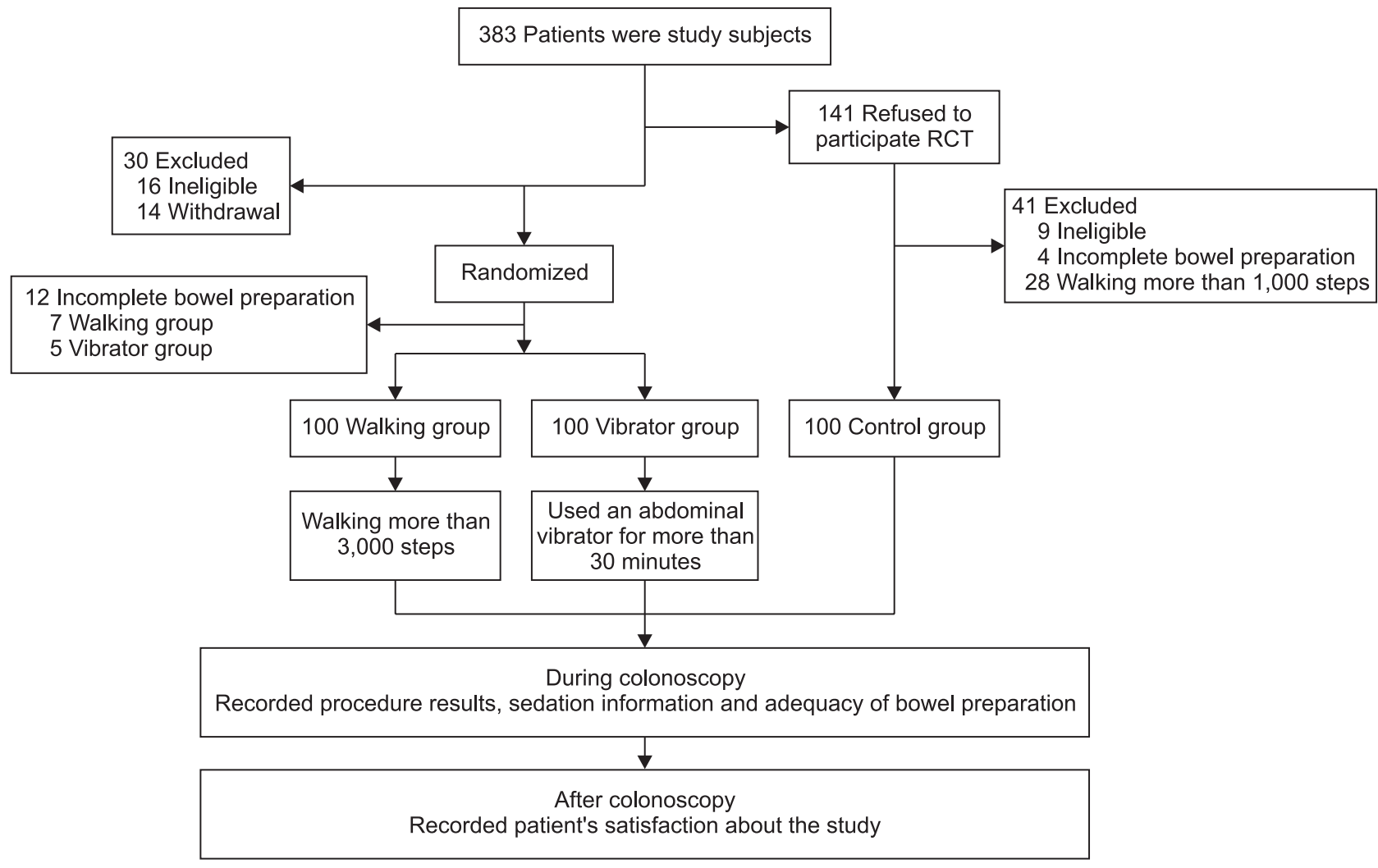

Fig. 1. Flow diagram of study enrollment, allocation, intervention, and evaluation. RCT, randomized controlled trial. 


\section{Bowel preparation for colonoscopy}

All patients consumed a liquid, low-fiber meal before taking the bowel preparation solution, which consisted of a 4-L PEG solution consumed as a split-dose from 18:00 to 21:00 on the day before the procedure and from 4:00 to 7:00 on the day of the procedure. The intervention (walking exercise or abdominal vibration stimulation) was scheduled to start not sooner than at 1 hour after taking the PEG solution to allow adequate gastric emptying and avoid nausea, vomiting, and aspiration of residual PEG solution from the stomach. Since the control group had no intervention (vibrator or walking), only the PEG solution was administered according to the schedule. All the patients enrolled in this study received intravenous fluid infusion immediately before the patients were moved to the endoscopic examination room. An overview of the timetable of patients during the period of preparation before colonoscopy is provided in Fig. 2.

Patients assigned to the walking group started walking 1 hour after taking the PEG solution on the day before the procedure, and were instructed to walk at least 3,000 steps freely before the procedure. These patients were given a pedometer to measure the number of steps walked. We did not set an upper limit on the number of steps for the walking group, and the patients began walking 1 hour after taking PEG on the day of the procedure. Walking could be performed up to an hour before the scheduled time to prepare for the procedure.

Patients in the vibrator group received abdominal vibration stimulation starting at 1 hour after taking the PEG solution on the day before procedure (three cycles, each consisting of 10 minutes of stimulation and 20 minutes of resting) and on the day of the procedure (up to five cycles). An abdominal vibration belt (CMT-03; Cozyma, Seoul, Korea) was used for abdominal vibration stimulation in this trial. The belt is freely sold on the market in the sector of dieting and fitness; as such a belt is not a medical device, it can be easily purchased. The patients were instructed to wear the belt on their abdomen, set the intensity at the lowest level, then turn on the belt and increase the intensity gradually (up to a maximum level of 6). The patients could reduce the stimulation intensity at their discretion. The attending nurse checked the patients' compliance with using the vibrating belt (Fig. 3). These patients were instructed to restrict their walking by staying in their room, and to report the number of cycles of vibration stimulation used. For the vibrator group, the vibrator application time was also limited to 1 hour before the procedure as with the walking group.

\section{On the day of the procedure}

The endoscopists were blinded to study allocation. The medication used for sedation was limited to midazolam, propofol, and meperidine. During the examination, the patient was

\section{Bowel preparation}

Reposing

Walking

Vibrator

Sleeping

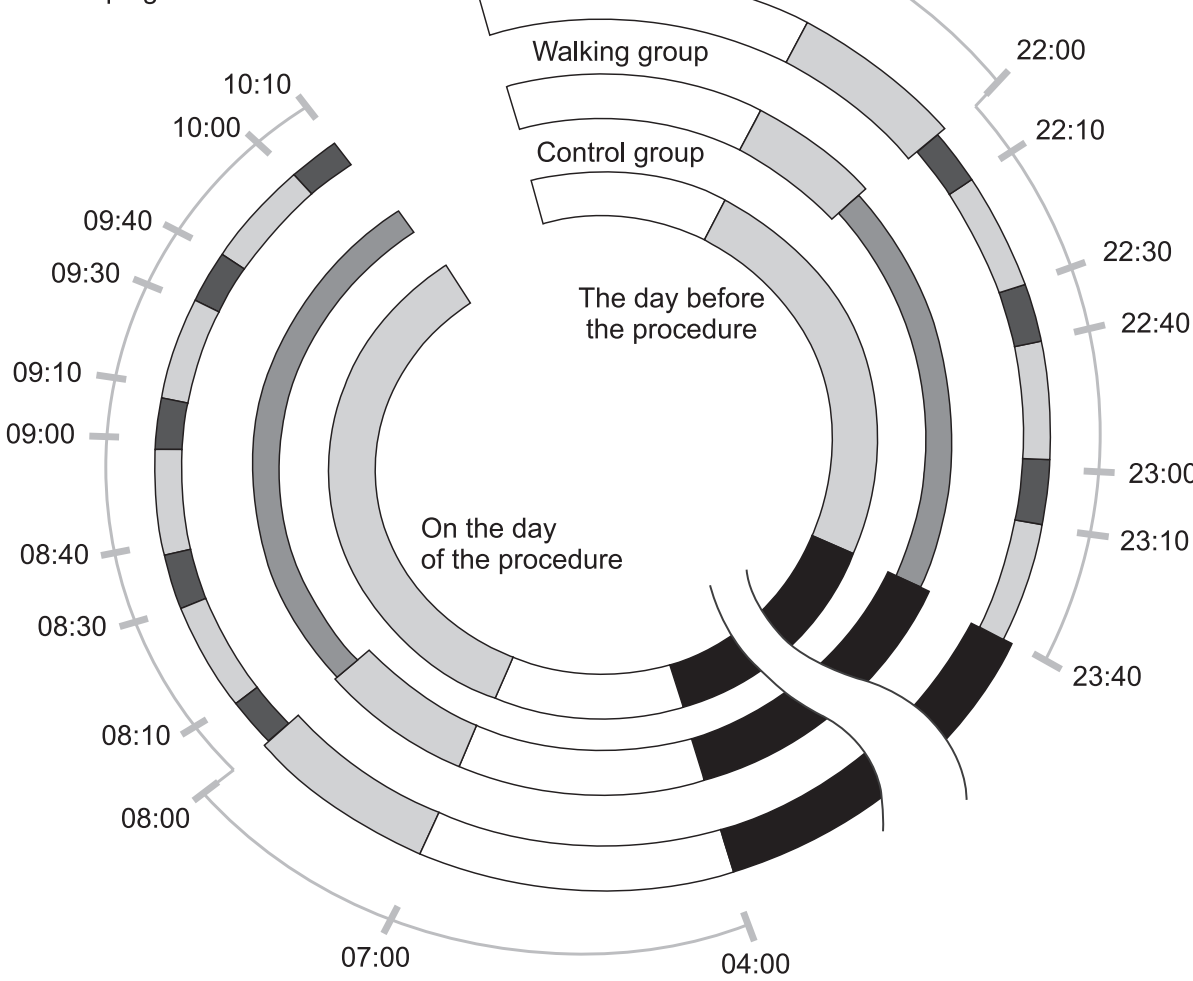

Fig. 2. Timetable of key activities before colonoscopy. Patients in the walking group walked at least 3,000 steps. Patients in the vibrator group received abdominal vibration stimulation. 
instructed to inform the nurse in the endoscopy room of any discomfort. When the patient complained of pain, the dose of medication was increased. After the colonoscopic procedure, endoscopists used a dedicated form to record the dose of the medication, cecal intubation time, scope withdrawal time, number of polyps, and adequacy of bowel preparation. The patients were returned to the ward by paramedics, where they received a questionnaire to assess patient tolerability (nausea and discomfort), patient satisfaction, and willingness to undergo the same bowel preparation protocol for a future colonoscopy. All the questions were related to the bowel preparation process, which is specified in the questionnaire. The questionnaire items were assessed on a scale from 0 to 5 ; for patient tolerability, a score of 0 indicated the lack of a symptom, while 5 indicated a severe symptom; for patient satisfaction, 0 indicated dissatisfaction, while 5 indicated high satisfaction.

\section{Assessment of bowel preparation}

During the procedure, two endoscopists blinded to study allocation evaluated the adequacy of bowel cleansing on the Boston Bowel Preparation Scale (BBPS). BBPS scores were determined by consensus between the two endoscopists and were reported on dedicated forms. The scores were reported separately for each segment of the colon (ascending, transverse, and descending), as well as for the entire colon, as follows: 0, unprepared colon segment with mucosa not seen because of solid stool that could not be cleared; 1 , portion of mucosa of the colon segment is seen; 2, minor amount of residual staining, small fragments of stool, and/or opaque liquid, but mucosa of the colon segment is seen well; 3, entire mucosa of the colon segment is seen well, with no residual staining, small fragments of stool, or opaque liquid. $^{10,11}$
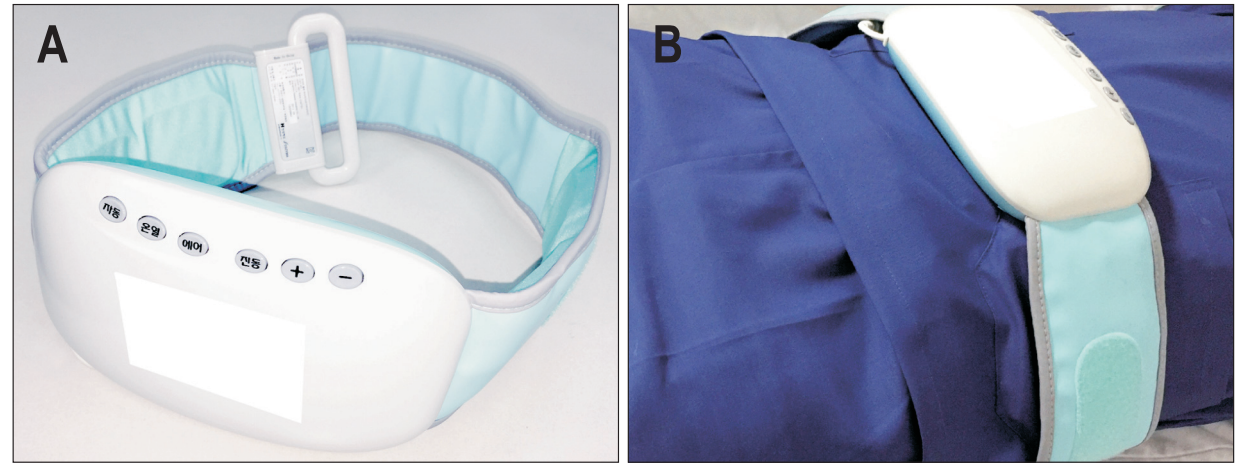

Fig. 3. Photographs of the vibration device and its mode of use. (A) Vibration belt. (B) Examples of how the vibration belt is worn by a patient lying in bed.

Table 1. Baseline Demographics of the Study Patients

\begin{tabular}{|c|c|c|c|c|}
\hline Characteristic & $\begin{array}{l}\text { Vibrator } \\
(\mathrm{n}=100)\end{array}$ & $\begin{array}{l}\text { Walking } \\
(\mathrm{n}=100)\end{array}$ & $\begin{array}{l}\text { Control } \\
(\mathrm{n}=100)\end{array}$ & $\mathrm{p}$-value \\
\hline Age, yr & $54.20 \pm 11.41$ & $53.79 \pm 12.70$ & $56.25 \pm 11.77$ & 0.297 \\
\hline Male sex & 66 & 75 & 72 & 0.363 \\
\hline BMI, $\mathrm{kg} / \mathrm{m}^{2}$ & $24.60 \pm 3.16$ & $25.30 \pm 3.99$ & $24.71 \pm 2.94$ & 0.145 \\
\hline \multicolumn{5}{|l|}{ Medical history } \\
\hline Hypertension & 38 & 28 & 30 & 0.278 \\
\hline Diabetes mellitus & 11 & 15 & 16 & 0.562 \\
\hline Old CVA & 3 & 1 & 0 & 0.171 \\
\hline Previous abdominal operation & 5 & 10 & 6 & 0.501 \\
\hline Physical activity* & 41 & 37 & 44 & 0.587 \\
\hline Constipation & 14 & 23 & 16 & 0.300 \\
\hline \multicolumn{5}{|l|}{ Smoking history } \\
\hline Smoker & 38 & 45 & 40 & 0.291 \\
\hline Smoking exposure, pack-years & $21.30 \pm 12.32$ & $20.30 \pm 12.46$ & $19.38 \pm 5.97$ & 0.415 \\
\hline Family history of colon cancer & 3 & 5 & 0 & 0.088 \\
\hline ASA physical status, $1 / 2$ & $56 / 44$ & $67 / 33$ & $71 / 29$ & 0.076 \\
\hline
\end{tabular}

Data are presented as mean \pm SD or number.

BMI, body mass index; CVA, cerebrovascular accident; ASA, American Society of Anesthesiologists.

*Moderate intensity exercise for $>30$ minutes more than three times per week. 


\section{Study outcomes and data collection}

The main outcome was adequacy of bowel cleansing, that is, BBPS score, which was compared among the three groups. The secondary outcomes were the factors affecting the main outcome (degree of bowel preparation), namely patients' compliance, as well as the cecal intubation time, which were also compared among the three groups. Demographic information was collected by the researchers via interviews with the patients. Data regarding bowel preparation was reported as follows: the ward nurse used a special form to record the time to first diarrhea and the total instances of diarrhea before the procedure; data regarding number of steps walked and cycles of abdominal stimulation were reported by the patients. Data regarding procedure characteristics and outcomes were reported by the endoscopists after the procedure. Data on patient tolerability and satisfaction were obtained by the researchers using questionnaires.

\section{Statistical analysis}

As we could not estimate the effect size from similar studies in the past, our sample size was based on the results from a pilot study that was performed in our center ( $n=30$ per group). It was estimated that the efficacy for good bowel preparation success would be $86 \%$ with vibrator application and $82 \%$ with walking exercise. Sample size of at least 88 patients was required for each intervention group to detect a difference in treatment success of a 5\% type-I error rate and 80\% power for a two-tailed chi-square test. On the basis of these results, 100 patients each were enrolled in the walking, vibrator, and control groups.

Continuous variables were expressed as mean \pm standard deviation and compared with the Student t-test. Categorical variables were expressed as total number and percentages. Oneway analysis of variance with post hoc analysis by the Bonferroni method was used to assess differences in continuous and ordinal variables among the groups. Statistical analyses were performed using the SPSS software package version 23.0 (IBM Corp., Armonk, NY, USA). p-values $<0.05$ were considered to indicate statistical significance.

\section{RESULTS}

\section{Baseline characteristics}

Three hundred and eighty-three patients were diagnosed as having colonic polyps and were admitted to our center for therapeutic endoscopy. Among them, 141 patients refused to participate in this randomized study, and they were enrolled

Table 2. Data Regarding Bowel Preparation and Colonoscopy

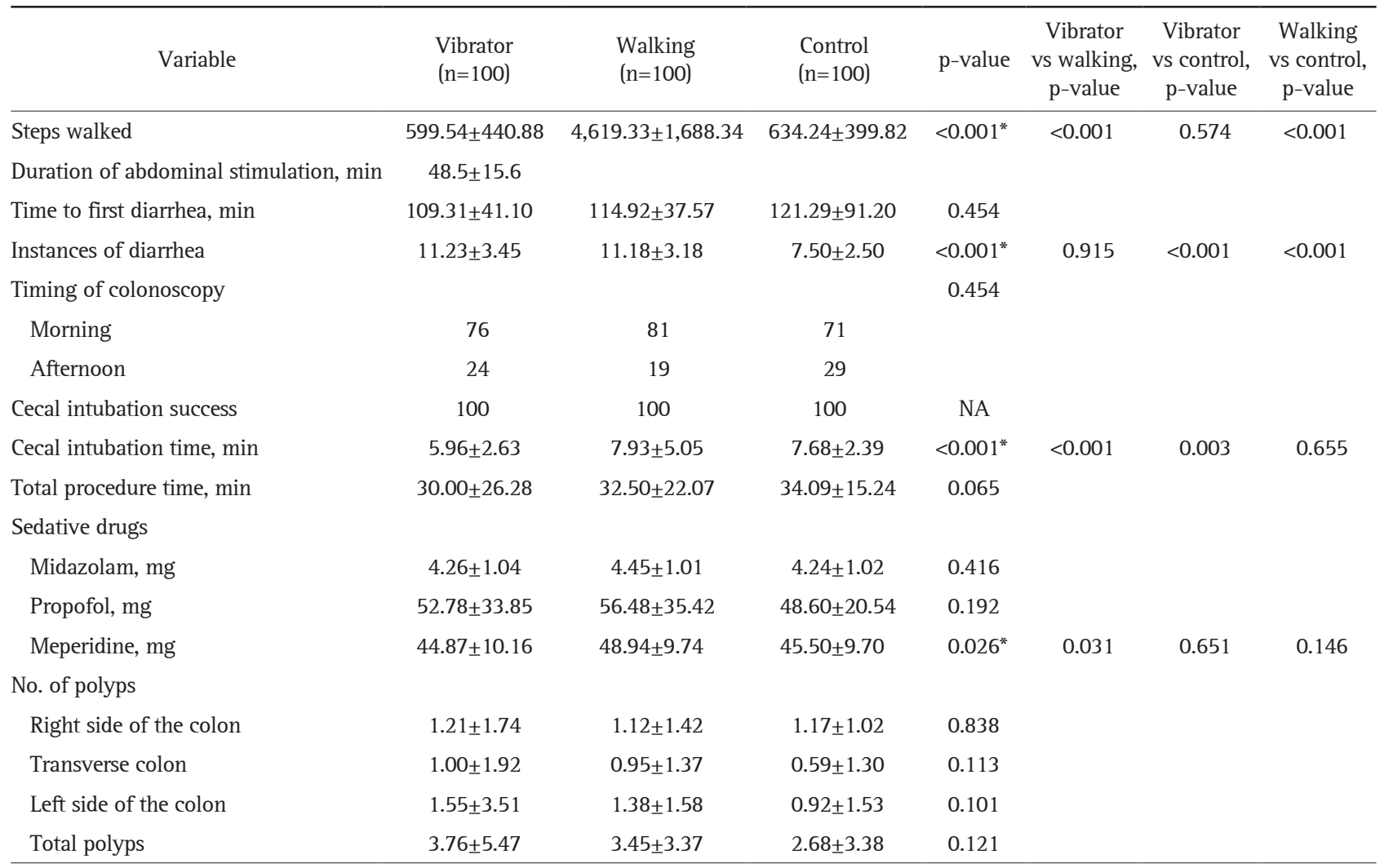

Data are presented as mean \pm SD or number.

NA, not applicable.

*Correction with Bonferroni post hoc. 
in the control group $(\mathrm{n}=100)$. Of the 242 patients who agreed to participate in the study, 42 patients were excluded, and the remaining 200 patients were randomized to the walking group or vibrator group (Fig. 1). Baseline characteristics including age, sex, body mass index, medical history, social history, and history of previous abdominal operation were not significantly different among the groups. Additionally, there was no significant difference in terms of physical exercise levels, incidence of constipation, family history of colon cancer, or American Society of Anesthesiologists physical status (Table 1).

\section{Details regarding bowel preparation and colonoscopy}

During preparation for colonoscopy, patients in the walking group walked 4,619.33 $\pm 1,688.34$ steps (walking group vs control group: $4,619.33 \pm 1,688.34$ vs $634.24 \pm 399.82$, $\mathrm{p}<0.001)$. There was no difference in the number of steps between the vibrator group and control group $(599.54 \pm 440.88$ vs $634.24 \pm 399.82, p=0.574)$. Patients in the vibrator group received abdominal vibration stimulation for $48.5 \pm 15.6$ minutes. There was also no difference among the three groups regarding the time to first diarrhea (vibrator group vs walking group vs control group: $109.31 \pm 41.10$ minutes vs $114.92 \pm 37.57$ minutes vs $121.29 \pm 91.20$ minutes, $p=0.454)$. However, both vibrator and walking group had significantly more instances of diarrhea than in the control group $(11.23 \pm 3.45$ vs $11.18 \pm 3.18$ vs $7.50 \pm 2.50$, $\mathrm{p}<0.001)$. Additionally, cecal intubation time was significantly shorter in the vibrator group than in the other groups $(5.96 \pm 2.63$ minutes vs $7.93 \pm 5.05$ minutes vs $7.68 \pm 2.39$ minutes, $\mathrm{p}<0.001$ ). Regarding sedative medication, the groups did not differ significantly regarding the total dose of midazolam or propofol, but patients in the vibrator group required a significantly lower dose of meperidine compared with walking group $(44.87 \pm 10.16$ mg vs $48.94 \pm 9.74 \mathrm{mg}, \mathrm{p}=0.031$ ) (Table 2 ).

\section{Adequacy of bowel cleansing}

The three groups were compared in terms of the BBPS score and distribution of scores for each colon segment and the entire colon. The total BBPS score did not differ significantly between the vibrator group and walking group $(7.38 \pm 1.55$ vs $7.39 \pm 1.55$, $\mathrm{p}=0.297$ ), and excellent bowel cleansing (BBPS score $\geq 8$ ) was

Table 3. Efficacy of Bowel Cleansing Assessed in Terms of the BBPS Score

\begin{tabular}{|c|c|c|c|c|c|c|c|}
\hline Colon segment & $\begin{array}{l}\text { Vibrator } \\
(n=100)\end{array}$ & $\begin{array}{l}\text { Walking } \\
(n=100)\end{array}$ & $\begin{array}{l}\text { Control } \\
(n=100)\end{array}$ & p-value & $\begin{array}{c}\text { Vibrator } \\
\text { vs walking, } \\
\text { p-value }\end{array}$ & $\begin{array}{l}\text { Vibrator } \\
\text { vs control, } \\
\text { p-value }\end{array}$ & $\begin{array}{c}\text { Walking } \\
\text { vs control, } \\
\text { p-value }\end{array}$ \\
\hline Transverse colon & $2.61 \pm 0.55$ & $2.48 \pm 0.67$ & $2.08 \pm 0.46$ & $<0.001^{*}$ & 0.120 & $<0.001$ & $<0.001$ \\
\hline Left side of the colon & $2.50 \pm 0.60$ & $2.47 \pm 0.64$ & $2.16 \pm 0.55$ & $<0.001^{*}$ & 0.705 & $<0.001$ & $<0.001$ \\
\hline \multicolumn{8}{|l|}{ Entire colon } \\
\hline Total BBPS score & $7.38 \pm 1.55$ & $7.39 \pm 1.55$ & $6.17 \pm 1.15$ & $<0.001^{*}$ & 0.297 & $<0.001$ & $<0.001$ \\
\hline BBPS score 0-5 & $8(8)$ & $15(15)$ & $22(22)$ & & & & \\
\hline BBPS score 6-7 & $36(36)$ & 35 (35) & $66(66)$ & & & & \\
\hline BBPS score 8-9 & $56(56)$ & $50(50)$ & $12(12)$ & & & & \\
\hline
\end{tabular}

Data are presented as mean \pm SD or number (\%). The Boston Bowel Preparation Scale (BBPS) score was assigned as follows: 0, unprepared colon segment with mucosa not seen because of solid stool that could not be cleared; 1 , portion of mucosa of the colon segment is seen; 2 , minor amount of residual staining, small fragments of stool, and/or opaque liquid, but mucosa of the colon segment is seen well; 3, entire mucosa of the colon segment is seen well, with no residual staining, small fragments of stool, or opaque liquid.

*Correction with Bonferroni post hoc.

Table 4. Comparison of Tolerability and Post-Procedure Satisfaction

\begin{tabular}{lcccc}
\hline \multicolumn{1}{c}{ Item } & Vibrator $(\mathrm{n}=100)$ & Walking $(\mathrm{n}=100)$ & Control $(\mathrm{n}=100)$ & $\mathrm{p}$-value \\
\hline Nausea* $^{*}$ & $0.73 \pm 0.96$ & $0.76 \pm 0.98$ & $0.81 \pm 0.58$ & 0.758 \\
General discomfort $^{*}$ & $0.74 \pm 0.97$ & $0.83 \pm 1.03$ & $0.73 \pm 0.55$ & 0.447 \\
Satisfaction with the procedure $^{\dagger}$ & $4.25 \pm 0.83$ & $4.21 \pm 0.82$ & $4.11 \pm 0.86$ & 0.125 \\
Willingness to repeat the protocol & $89(89)$ & $95(95)$ & NA & 0.191 \\
Adverse event & $2(2)^{\ddagger}$ & 0 & NA & 0.497 \\
\hline
\end{tabular}

Data are presented as mean \pm SD or number (\%).

NA, not applicable.

*Patient tolerability was measured on a scale from 0 to 5 , with 0 indicating the symptom did not occur and 5 indicating the symptom was severe; ${ }^{\dagger}$ Patient satisfaction was measured on a scale from 0 to 5 , with 0 indicating complete dissatisfaction and 5 indicating high satisfaction; ${ }^{\ddagger} 0$ ne patient complained of itching sensation on the skin under the belt, and another patient complained of mild abdominal discomfort. 
noted in approximately half of patients in each group (56\% vs $50 \%, p=0.799$ ). There were no significant between-group differences in the BBPS score for the ascending colon $(2.38 \pm 0.67$ vs $2.36 \pm 0.71, \mathrm{p}=0.959)$, transverse colon $(2.61 \pm 0.55$ vs $2.48 \pm 0.67$, $\mathrm{p}=0.120)$, or descending colon $(2.50 \pm 0.60$ vs $2.47 \pm 0.64$, $\mathrm{p}=0.705$ ). However, both vibrator and walking groups had a significantly higher BBPS score than that of the control group for all segments of the colon and the entire colon $(p<0.001)$ (Table 3$)$. Additionally, the percentage of poor bowel preparations (BBPS score $\leq 5$ ) in the control group was higher than that in the two groups (vibrator group vs walking group vs control group: $8 \%$ vs $15 \%$ vs $22 \%, \mathrm{p}=0.029$ ).

\section{Patient tolerability and satisfaction}

After the procedure, the incidence and severity of nausea, the frequency and severity of general discomfort, the degree of satisfaction with the procedure, and the willingness to undergo the same bowel preparation protocol for a future colonoscopy were assessed using patient-reported questionnaires. There was no statistically significant difference among the three groups. Only two patients who used the vibration belt reported adverse effects, whereas no patients who performed walking exercise reported adverse effects (Table 4).

\section{DISCUSSION}

This was the first study to depict the validity and usefulness of abdominal vibration stimulation versus walking exercise to improve the quality of bowel preparation for colonoscopy. This prospective, randomized study showed that patients who received abdominal vibration stimulation had adequate bowel clearance, and most clinical indicators were similar between the vibrator group and the walking group.

While bowel preparation protocols have improved substantially over the years, bowel cleansing for colonoscopy is difficult in the patients who are unable or unwilling to perform walking exercise during the preparation period. To address this limitation, we explored the effectiveness of abdominal physical stimulation to involve constipation, a condition frequently encountered in this patient population. The mechanism by which abdominal physical stimulation reduces constipation is presumed to involve activation of stretch receptors, which can reinforce the gastrocolic reflex, trigger intestinal and rectal muscle contraction, and stimulate somato-autonomic reflexes. ${ }^{12,13}$ In the GI tract, parasympathetic stimulation increases GI motility, increases secretion, and relaxes the sphincters. ${ }^{14}$ Physical stimulation of the abdomen induces a parasympathetic division of the GI tract, which stimulates motility. ${ }^{14}$ In contrast to laxatives, abdominal massage has been reported to help improve constipation by increasing GI motility with few side effects. ${ }^{8,9,15}$ In our study, patients reported no substantial discomfort during vibration stimulation, and excellent bowel cleansing was noted on colonoscopy despite the fact that vibration stimulation was applied for a relatively short time (10 minutes per cycle, up to eight cycles in total).

In combination with abdominal massage, whole-body vibration and abdominal vibration stimulation can stimulate peristalsis, decrease colonic transit time, increase the frequency of bowel movements, and decrease discomfort in constipated patients. ${ }^{8,9}$ Therefore, we assume that abdominal vibration stimulation and walking exercise may achieve a similar physiological effect and thus clinical relevance for bowel cleansing before colonoscopy. In our study, the BBPS score and total score of the vibrator group were better than those of the control group in all segments. In particular, the percentage of poor bowel preparations with a BBPS score less than five ${ }^{10}$ was $8 \%$, which was the lowest among the three groups. In addition, this is a significantly lower rate than that in the control group $(p=0.033$, data was not shown). The walking group, which performed intensive walking (more than 3,000 steps), also showed a higher BBPS score than that of the control group. Although intensive walking leads to a good quality of bowel preparation, it is relatively difficult for patients with gait disturbance, and bowel cleansing with abdominal vibration stimulation is relatively easy and was found to show equivalent bowel preparation results to that of walking over 3,000 steps in our study.

In the present study, the vibrator group and the walking group showed the same outcomes regarding most clinical aspects. While there was no difference in BBPS scores (adequacy of bowel cleansing), cecal intubation time was significantly shorter in the vibrator group $(5.96 \pm 2.63$ vs $7.93 \pm 5.05, p<0.001)$. On the other hand, there was no difference in cecal intubation time between the walking and control groups $(7.93 \pm 5.05$ vs $7.68 \pm 2.39, p=0.655)$. Vibration stimulation before colonoscopy seems to help facilitate endoscope insertion by regulation of colon motility, although the exact mechanism underlying this effect is not known. Additionally, BBPS uses a summation score to assess bowel preparation quality in three segments of the colon after all cleansing maneuvers during colonoscopy. ${ }^{16}$ Thus, there could be some differences between bowel cleansing scores on colonoscope insertion and withdrawal. In this study, we found that BBPS scores measured during colonoscope withdrawal in both vibrator and walking groups were not different; this was possibly due to vibration stimulations that might improve the fluid retention during the colonoscopy insertion. Therefore, cecal intubation time was estimated to be shorter in the vibrator group than in other groups. In a prospective study of 693 outpatients, poor bowel preparation was identified among the factors associated with delayed cecal intubation. ${ }^{4,17}$ However, in our study, there was no difference in cecal intubation between the walking and control groups, even though bowel preparation was good. Poor bowel preparation may affect the cecal intubation time, but among expert colonoscopists, bowel preparation is of less concern when the mean BBPS score 
is greater than 6 , as scores greater than 6 indicate good bowel preparation.

In this study, the BBPS score was somewhat higher than that reported in a previous study that compared types of solutions for bowel preparation. In a study of 663 patients who underwent screening colonoscopy, the average BBPS score was 6.2. In a study of 92 patients in whom the availability of an educational video on diet was evaluated before outpatient colonoscopy, the mean BBPS score of both groups was 7.0 (video group vs no video group: 7.0 vs 7.0, $\mathrm{p}=0.45) .{ }^{18}$ A comparison of two intensive bowel cleansing regimens in patients with poor bowel preparation showed lower mean values than that found in our study, with 7.04 in group 1 (4-L split-dose of a PEG regimen) and 6.43 in group 2 (2-L split-dose of a PEG plus ascorbic acid regimen, $\mathrm{p}=0.05){ }^{19}$ In our study, BBPS scores higher than those reported in previous studies were observed as a result of additional efforts, such as the walking exercise and vibration stimulation, in addition to the use of the PEG solution. Meanwhile, the BBPS score of control group was $6.17 \pm 1.15$, which is similar to the results of previous studies. ${ }^{4}{ }^{49}$ Additionally, it was also presumed that patients' willingness to undergo bowel preparation was higher because the procedure was for therapeutic purposes rather than for screening purposes and they understood the importance of bowel preparation for the success of a therapeutic procedure.

There were some limitations to our study. First is the lack of a randomized control group. Second, the enrolled patients were all hospitalized for endoscopic resection and not screening colonoscopy. Third, because only therapeutic colonoscopy was analyzed, the relationship between bowel preparation quality and the number of polyps is unclear. Most polyps had already been confirmed during prior examination at the local clinic. Therefore, we could not evaluate polyp and adenoma detection rates. Fourth, there are no guidelines regarding the optimal duration of stimulation, and no conclusion in this direction could be drawn based on our data because the protocol allowed various durations of stimulation (30 to 80 minutes) at the patient's discretion, and we found no significant correlation between the duration of stimulation and the BBPS scores. Further study is needed to determine the optimal duration of abdominal vibration stimulation for bowel preparation. Fifth, not all colonoscopy procedures were performed in the morning. The time interval between bowel preparation and colonoscopy is an important factor for procedure success. In our study, further analysis of the relationship between time interval and the adequacy of bowel preparation was not possible because a few patients underwent colonoscopy in the afternoon. Sixth, we did not use small-volume (2-L PEG) as a bowel cleansing solution but instead used 4-L PEG, because 4-L PEG is used in our center guideline for therapeutic colonoscopy in admitted patients. We will perform a future study using a small-volume solution to relieve bowel cleansing discomfort. Finally, patients in this study were relatively lean, with a mean body mass index of around $25 \mathrm{~kg} / \mathrm{m}^{2}$. Moreover, all subjects were able to perform walking exercise. Therefore, the results do not fully reflect the efficacy of bowel preparation in patients with disabilities resulting in gait disturbance.

In conclusion, this is the first study to demonstrate that bowel preparation accompanied with abdominal vibration stimulation show results similar to those obtained with walking exercise. Our findings suggest that bowel preparation with abdominal vibration stimulation may help achieve adequate bowel cleansing in patients who are unable or unwilling to perform walking exercise or patients with nervous system diseases who may be vulnerable to lengthy sedation. However, further study is needed to validate our findings in this population.

\section{CONFLICTS OF INTEREST}

No potential conflict of interest relevant to this article was reported.

\section{AUTHOR CONTRIBUTIONS}

Planning the study design: C.K.N., I.S.K., S.G.L, J.H.K. Reviewing the data: C.K.N. Analyzing the data: C.K.N., E.L., B.P., J.H.K Drafting the manuscript: C.K.N., K.M.L. Collecting the data: I.S.K. G.H.L., J.W.P. Interpreting the data: E.L., B.P., S.J.S. Nursing enrolled patients: H.J.H. Developing the study concept: S.G.L., S.J.S Critically revising the manuscript: K.M.L. All authors approved the final version of the article and agree to be accountable for all aspects of the work.

\section{ORCID}

Choong-Kyun Noh https://orcid.org/0000-0002-3607-8120 In Sung Kim https://orcid.org/0000-0002-3587-9735 Gil Ho Lee https://orcid.org/0000-0001-7695-0828 Jin Woong Park https://orcid.org/0000-0001-8132-2789 Eunyoung Lee https://orcid.org/0000-0002-9440-9119 https://orcid.org/0000-0002-5271-1571 https://orcid.org/0000-0002-5053-7522 https://orcid.org/0000-0003-2045-5099 https://orcid.org/0000-0003-1849-4435 https://orcid.org/0000-0002-3352-5079 https://orcid.org/0000-0003-3785-693X

\section{REFERENCES}

1. Hendry PO, Jenkins JT, Diament RH. The impact of poor bowel preparation on colonoscopy: a prospective single centre study of 10,571 colonoscopies. Colorectal Dis 2007;9:745-748.

2. Chokshi RV, Hovis CE, Hollander T, Early DS, Wang JS. Prevalence of missed adenomas in patients with inadequate 
bowel preparation on screening colonoscopy. Gastrointest Endosc 2012;75:1197-1203.

3. Lebwohl B, Kastrinos F, Glick M, Rosenbaum AJ, Wang T, Neugut AI. The impact of suboptimal bowel preparation on adenoma miss rates and the factors associated with early repeat colonoscopy. Gastrointest Endosc 2011;73:1207-1214.

4. Kim WH, Cho YJ, Park JY, Min PK, Kang JK, Park IS. Factors affecting insertion time and patient discomfort during colonoscopy. Gastrointest Endosc 2000;52:600-605.

5. Kim HS, Park DH, Kim JW, et al. Effectiveness of walking exercise as a bowel preparation for colonoscopy: a randomized controlled trial. Am J Gastroenterol 2005;100:1964-1969.

6. Lämås K, Lindholm L, Stenlund H, Engström B, Jacobsson C. Effects of abdominal massage in management of constipation: a randomized controlled trial. Int J Nurs Stud 2009;46:759-767.

7. Lämås K, Graneheim UH, Jacobsson C. Experiences of abdominal massage for constipation. J Clin Nurs 2012;21:757-765.

8. Mimidis K, Galinsky D, Rimon E, Papadopoulos V, Zicherman Y, Oreopoulos D. Use of a device that applies external kneading-like force on the abdomen for treatment of constipation. World J Gastroenterol 2005;11:1971-1975.

9. Wu TJ, Wei TS, Chou YH, et al. Whole-body vibration for functional constipation: a single-centre, single-blinded, randomized controlled trial. Colorectal Dis 2012;14:e779-e785.

10. Lai EJ, Calderwood AH, Doros G, Fix OK, Jacobson BC. The Boston Bowel Preparation Scale: a valid and reliable instrument for colonoscopy-oriented research. Gastrointest Endosc 2009;69 (3 Pt 2):620-625.

11. Kim EJ, Park YI, Kim YS, et al. A Korean experience of the use of Boston Bowel Preparation Scale: a valid and reliable instrument for colonoscopy-oriented research. Saudi J Gastroenterol 2014;20:219-224.

12. Brookes SJ, Chen BN, Costa M, Humphreys CM. Initiation of peristalsis by circumferential stretch of flat sheets of guinea-pig ileum. J Physiol 1999;516 (Pt 2):525-538.

13. Liu Z, Sakakibara R, Odaka T, et al. Mechanism of abdominal massage for difficult defecation in a patient with myelopathy (HAM/TSP). J Neurol 2005;252:1280-1282.

14. Moyer CA, Rounds J, Hannum JW. A meta-analysis of massage therapy research. Psychol Bull 2004;130:3-18.

15. Ernst E. Abdominal massage therapy for chronic constipation: a systematic review of controlled clinical trials. Forsch Komplementarmed 1999;6:149-151.

16. ASGE Standards of Practice Committee, Saltzman JR, Cash BD, et al. Bowel preparation before colonoscopy. Gastrointest Endosc 2015;81:781-794.

17. Bernstein C, Thorn M, Monsees K, Spell R, O’Connor JB. A prospective study of factors that determine cecal intubation time at colonoscopy. Gastrointest Endosc 2005;61:72-75.

18. Rice SC, Higginbotham T, Dean MJ, Slaughter JC, Yachimski PS, Obstein KL. Video on diet before outpatient colonoscopy does not improve quality of bowel preparation: a prospective, randomized, controlled trial. Am J Gastroenterol 2016;111:1564-1571.

19. Gimeno-García AZ, Hernandez G, Aldea A, et al. Comparison of two intensive bowel cleansing regimens in patients with previous poor bowel preparation: a randomized controlled study. Am J Gastroenterol 2017;112:951-958. 